\title{
Vigilância pós-alta e o seu impacto na incidência da infecção do sítio cirúrgico*
}

\author{
POST-DISCHARGE SURVEILLANCE AND ITS IMPACT ON \\ SURGICAL SITE INFECTION INCIDENCE
}

VIGILANCIAPOS-ALTAY SU IMPACTO EN LAINCIDENCIADE LAINFECCIÓN DEL SITIO QUIRÚRGICO

\author{
Adriana Cristina Oliveira', Suely Itsuko Ciosak", Claudia D'Lorenzo"I"
}

\section{RESUMO}

As infecções do sítio cirúrgico (ISC) por sua elevada incidência e repercussões associadas, representam importante problema dentre as infecções hospitalares. Objetivou-se neste estudo determinar a incidência da ISC de pacientes submetidos à cirurgia para obesidade mórbida (COM) e gástrica por outras causas (CGOC), durante a internação e após alta. Foram acompanhados em dois hospitais terciários, de ensino da cidade de São Paulo, entre agosto de 2001 e março de 2002, conforme metodologia NISS, 158 pacientes, sendo 81 submetidos à COM e 77 à CGOC, por um período de 30 dias. Notificou-se 64 ISC, 6,3\% durante a internação e $34,1 \%$ após a alta. Durante a internação, a incidência de ISC foi de 5,0\% no grupo COM e de 7,8\% no CGOC e com a vigilância pósalta, estas taxas aumentaram para $55,6 \%$ e $24,7 \%$, respectivamente, demonstrando que vigilância pós-alta no pacientes cirúrgico é uma importante ferramenta para obter dados confiáveis e redirecionar as políticas de prevenção e controle da ISC.

\section{DESCRITORES}

Controle de infecções.

Infecção hospitalar.

Obesidade mórbida.

Cirurgia bariátrica.

\section{ABSTRACT}

Because of their high incidence and associated repercussions, Surgical Site Infections (SSI) are an important problem among hospital-acquired infections. The aim of this study was to determine the incidence of SSIs in patients submitted to morbid obesity and gastric surgeries for other causes during the hospitalization and post-discharge periods. In this survey, conducted in two teaching hospitals in the city of São Paulo between August of 2001 and March of 2002, in accordance to the NISS methodology, 158 patients were followed - 81 had been submitted to morbid obesity surgeries and 77 to gastric surgeries for other causes. In 64 cases there occurred SSI, of which $6.3 \%$ during hospitalization and $34.1 \%$ after discharge. During hospitalization, the incidence of SSI was $5 \%$ in the morbid obesity surgeries group and $7.8 \%$ in the gastric surgeries for other causes group; in the post-discharge period, the figures increased to $55.5 \%$ and $24.7 \%$ respectively. The-se results confirm the importance of following surgical patients after discharge and demonstrate that post-discharge surveillance is an important tool for obtaining dependable data and redirect SSI's prevention and control policies.

\section{KEY WORDS}

Infection control.

Cross infection.

Obesity morbid.

Bariactric surgery.

\section{RESUMEN}

Las infecciones del sitio quirúrgico (ISC) por su elevada incidencia y repercusiones asociadas, representan importante problema entre las infecciones hospitalarias. Fue objetivado en este estudio determinar la incidencia de la ISC de pacientes sometidos a la cirugía para obesidad mórbida (COM) e gástrica por otras causas (CGOC), durante la internación y pos alta. Fueron acompañados en dos hospitales terciarios, de enseñanza de la ciudad de São Paulo, entre agosto de 2001 y marzo de 2002, según metodología NISS, 158 pacientes, siendo 81 sometidos a la COM e 77 a la CGOC, por un periodo de 30 días. Se notificó 64 ISC, 6,3\% durante la internación y 34,1\% después del alta. Durante la internación, la incidencia de ISC fue de 5,0\% en el grupo COM y de $7,8 \%$ en el CGOC y con la vigilancia pos-alta, estas tasas aumentaron para 55,6\% y $24,7 \%$, respectivamente, demostrando que vigilancia pos-alta en los pacientes quirúrgicos es una importante herramienta para obtener datos confiables y reencaminar las políticas de prevención y control de la ISC.
* Extraído da tese de doutorado "Infecção do sítio cirúrgico em pacientes submetidos à cirurgia do aparelho digestivo: uma proposta de predição de risco", Escola de Enfermagem, Universidade de São Paulo, 2003.

I Enfermeira. Mestre e Doutora. Professora da Escola de Enfermagem, Universidade Federal de Minas Gerais (UFMG), Belo Horizonte, MG, Brasil.

adriana@enf.ufmg.br

II Enfermeira.Doutora. Professora Livre Docente da Escola de Enfermagem, Universidade de São Paulo (EEUSP), São Paulo, SP, Brasil. siciosak@usp.br

III Médica da Fundação Nacional de Saúde (FUNASA). Doutora em Epidemiologia pela Faculdade de Medicina, Universidade Federal de Minas Gerais (UFMG), Belo Horizonte, MG, Brasil.

\section{DESCRIPTORES}

Control de infecciones.

Infección hospitalaria.

Obesidad mórbida.

Cirugía bariatrica. 


\section{INTRODUÇÃO}

As infecções de sítio cirúrgico (ISC) são aquelas que ocorrem na incisão cirúrgica, acometendo tecidos, órgãos e cavidades manipulados durante a operação, podendo ser diagnosticadas até 30 dias após a data de realização do procedimento. Na grande maioria dos hospitais a ISC constitui o primeiro ou segundo sitio mais importante de infecção sendo algumas vezes superada apenas pela infecção do trato urinário ${ }^{(1-2)}$.

A ISC é uma complicação relevante, por contribuir para o aumento da mortalidade e morbidade dos pacientes póscirúrgicos, causando prejuízos físicos, emocionais, como seu afastamento do trabalho e do convívio social. Além disso, eleva consideravelmente os custos com o tratamento, repercutindo também em uma maior permanência hospitalar. Apesar de ser a complicação mais comum de uma cirurgia, a ISC deve ser evitada e a sua ocorrência deve estar dentro dos níveis aceitos pelos órgãos competentes ${ }^{(1-3)}$.

De acordo com o Ministério da Saúde todos os hospitais devem possuir diretrizes e normas para a prevenção e controle das infecções hospitalares, organizadas através de Programas de Controle de Infecção Hospitalar (PCIH), desenvolvidos pelas Comissões de Controle de Infecção Hospitalar (CCIH) ${ }^{(4)}$.

Para tanto, é de competência da CCIH realizar a vigilância epidemiológica de todos os pacientes, principalmente daqueles que apresentam um risco maior para infecção, como aqueles submetidos a cirurgia. É ainda sugerido que, essa vigilância deva ocorrer por meio de métodos prospectivos, como a busca ativa, sistemática e contínua das infecções, bem como de sua distribuição ${ }^{(4)}$.

Nesse contexto percebe-se que, em relação a ISC, a vigilância do paciente cirúrgico na maioria das instituições, tem ocorrido apenas durante o período de internação. Entretanto, o Centro de Controle e Prevenção de Doenças (CDC) de Atlanta (Estados Unidos), órgão de referência para o controle e prevenção das infecções hospitalares, recomenda que no paciente cirúrgico, devido aos fatores específicos, inerentes ao ato cirúrgico e sua condição, a vigilância deva ser ampliada após a alta hospitalar, apontando para cifras em torno de $19 \%$ a $84 \%$ das ISC que se manifestam após a alta deste paciente ${ }^{(1,3,5-12)}$.

Desta forma, frente ao exposto, pode-se inferir que estudos que restringem o seguimento do paciente cirúrgico somente durante o período de internação tendem a apresentar menores taxas de ISC quando comparados àqueles que incluem o seguimento depois da alta.

Assim sendo e, tendo em vista a relevância epidemiológica da questão e a importância da vigilância dos pacientes cirúrgicos durante a internação e após a alta hospitalar, este trabalho tem como objetivo determinar a incidência da ISC em pacientes submetidos à cirurgia do aparelho digestivo por obesidade mórbida e cirurgia gástrica por outras causas (gastrectomia), durante a internação e após a alta, além de avaliar o impacto da vigilância pós-alta.

\section{MÉTODO}

Foi conduzido um estudo descritivo, comparando a ISC em dois grupos de pacientes: o primeiro submetido a cirurgia do aparelho digestivo por obesidade mórbida (COM) e o segundo submetido a cirurgia gástrica por outras causas (CGOC), em dois hospitais de ensino, de cuidados terciários, localizados na cidade de São Paulo.

Foram elegíveis pacientes submetidos à cirurgia de obesidade mórbida (COM) e gástrica por outras causas (CGOC) entre agosto de 2001 a março de 2002. Como critério de inclusão foi adotada a recomendação do National Nosocomial Infection Surveillance System (NISS), cujos critérios são: ter sido submetido a intervenção cirúrgica $\mathrm{COM}$ ou CGOC, permanência hospitalar superior a 24 horas (ou seja, a data de admissão e alta ocorreram em dias diferentes do calendário), procedimento cirúrgico que inclua incisão e sutura antes do paciente deixar o centro cirúrgico. Assim, os pacientes incluídos no estudo foram acompanhados desde o momento da admissão até a alta e por 30 dias depois da data da cirurgia ${ }^{(1)}$.

Durante a internação, foi realizada busca ativa diária das infecções do sítio cirúrgico nos pacientes incluídos no estudo e internados nas enfermarias da cirurgia do aparelho digestivo (CAD), por dois alunos de graduação previamente treinados e, devidamente supervisionados pela pesquisadora principal. A coleta de dados foi feita utilizando-se os prontuários do pacientes, registros médicos e de enfermagem e, quando necessário, avaliação direta da incisão cirúrgica.

Foram coletados dados referentes à identificação do paciente; sexo (masculino e feminino); idade ( $\leq 60$ e $>60$ anos), tempo de permanência hospitalar ( 0 a 2; 3 a 5 e $>5$ dias), duração da cirurgia (correspondendo ao valor em horas do tempo cirúrgico máximo de $75 \%$ das cirurgias analisadas no estudo NNIS= 3horas) classificação da cirurgia quanto ao potencial de contaminação (limpa, potencialmente contaminada, contaminada e infectada), tipo de cirurgia (urgência ou eletiva), condição clínica pré-operatória, presença de doença(s) de base, pela avaliação do anestesista através do escore ASA - American Society Anestesiolgy ( Asa 1: paciente saudável; Asa 2 paciente com doença sistêmica discreta, sem descompensação; Asa 3: paciente com doença sistêmica grave, com limitação da atividade e Asa 4 e 5 : 
paciente com doença sistêmica incapacitante e remota possibilidade de vida por mais de 24 horas $)^{(1-2)}$.

Para o diagnóstico da ISC, o CDC através do guideline de prevenção e controle da infecção do sitio cirúrgico, propõe que a presença da secreção purulenta deva ser considerada como padrão-ouro para a notificação da mesma, desde que não caracterize reação local ao ponto. E, ainda recomenda que, após a notificação, a ISC deva ser classificada de acordo com sua localização como: superficial (quando acomete apenas pele ou tecido celular subcutâneo), profunda (quando envolve estruturas profundas da parede, fáscia e camada muscular) e órgão/cavidade (quando envolve estruturas anatômicas, abertas ou manipuladas durante o ato cirúrgico $)^{(1)}$, critérios estes adotados neste estudo.

Na vigilância pós-alta considerou-se o seguimento dos pacientes de ambos os grupos (COM e CGOC) até o trigésimo dia, conforme recomendado pela metodologia $\mathrm{NISS}^{(1)}$.

A vigilância pós-alta foi feita através de contato telefônico e retorno ambulatorial, devido à diferença entre as duas instituições de origem dos grupos, em relação à centralização ou não dos retornos dos pacientes para controle médico. Ou seja, em uma instituição havia um ambulatório único com dias e horários fixos para o retorno dos pacientes para retirada de pontos e avaliação médica e, na outra, de acordo com o cirurgião, o paciente poderia retornar ao seu consultório, ou outros locais (unidades básicas de saúde, etc) com horários e turnos diversos independentes do controle da instituição.

Desta forma, os pacientes cirúrgicos da CGOC foram acompanhados no retorno ambulatorial, paralelamente ao retorno médico, a fim de se evitar um custo adicional para o paciente com o transporte. Este retorno ocorreu dentro de sete a dez dias da data da cirurgia ou em período superior a este em caso de maior permanência hospitalar. O paciente era atendido por um membro da equipe do estudo e este o avaliava destacando o local da incisão cirúrgica em relação a ocorrência de hiperemia, calor, rubor, deiscência, presença de secreção no local da incisão e se presente, seu aspecto coloração, localização e quantidade.

E, para os pacientes da COM, como o horário e diversidade dos ambulatórios do atendimento médico foi incompatível com os horários da equipe do estudo, optou-se pelo seguimento deste grupo, por contato telefônico, realizado dentro do mesmo intervalo em dias (sete a dez dias) e mesmos critérios propostos para o grupo anterior para as ligações.

Durante o contato telefônico de forma semelhante ao retorno ambulatorial o paciente foi questionado sobre sinais característicos de infecção na ferida operatória como: hiperemia, calor, rubor, deiscência, presença de secreção no local da incisão e aspecto da secreção, quando presente.
Cuidados adicionais foram tomados na formulação das perguntas para o contato telefônico a fim de se evitar a possibilidade de indução nas respostas. E, quando o paciente relatava a presença de secreção na ferida, de forma acessível the era solicitado a descrição desta com detalhamento do seu aspecto, coloração, localização e quantidade, visando aumentar a confiabilidade de sua resposta para notificação ou não da ISC. Após o contato todos os casos eram discutidos entre a equipe do estudo e quando necessário com a equipe assistente buscando a homogeneidade de critérios para notificação ou não dos casos.

Para análise, os dados foram coletados em instrumentos específicos, codificados para as diversas variáveis do estudo e digitados no Programa ACESS e, para o tratamento estatístico foram utilizados os programas EPI-INFO versão 6.0 e a versão 11.5 do SPSS. Para a comparação dos grupos e a incidência da ISC durante e após a alta foram utilizados análise univariada, descritiva simples (absoluta) e percentual.

\section{RESULTADOS}

No período de agosto de 2001 a março de 2002, foram acompanhados 158 prontuários de pacientes, em ambos os grupos (COM e CGOC), sendo 47,5\% (75) do sexo masculino e 52,5\% (83) do sexo feminino. A média de idade nos dois grupos foi de 45,4 anos.

Em relação à ocorrência da ISC foram diagnosticadas durante a internação 10 ISC, com a taxa de incidência de 6,3\%, considerando ambos os grupos do estudo (COM e CGOC).

Analisando-se a taxa de incidência da ISC, detectada durante o período de internação, na população específica de cada grupo, em pacientes submetidos a COM esta foi de 5,0\% (4/81) e em pacientes submetidos a CGOC de 7,8\% (6/77).

Com a vigilância pós-alta, foram notificados 41 (41/81) casos de ISC no grupo COM e 13 (13/77) no grupo CGOC, como mostra a Figura 1. Desta forma, com a realização da vigilância pós-alta verificou-se uma taxa global de 55,5\% e $24,7 \%$ de ISC para os grupos COM e CGOC. Um incremento da taxa de infecção em 11,1 e 3,2 vezes respectivamente.

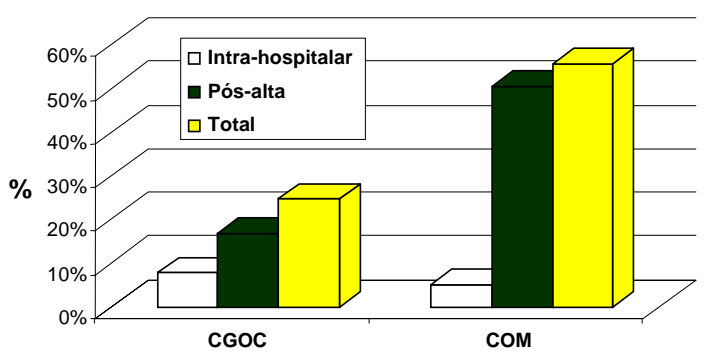

Figura 1 - Distribuição das Infecções do Sítio Cirúrgico durante a internação e após a alta - São Paulo - 2002 
Em relação as variáveis: tempo de permanência, potencial de contaminação, tipo de cirurgia (eletiva/urgência), duração da cirurgia, e ASA foi realizada uma análise univariada para cada um dos grupos COM e CGOC em relação a ocorrência ou não da ISC e o local do diagnóstico, não se evidenciando nenhuma associação entre eles.

Uma observação importante foi feita em relação à duração média dos procedimentos cirúrgicos realizados nos grupos CGOC e COM.

A duração de uma cirurgia é considerada prolongada, de acordo com a metodologia adotada neste estudo (NNIS) quando for maior do que aquela definida por $75,0 \%$ dos procedimentos semelhantes, realizados e analisados, comparativamente ${ }^{(13)}$. Assim sendo, de acordo com o ponto de corte NNIS, a duração prevista tanto para COM quanto para CGOC é de três horas ${ }^{(14)}$, e nesse estudo foi calculada de acordo com o tempo médio, em minutos, para cada procedimento e para cada grupo.

Verificou-se que, como média geral o grupo CGOC (147 minutos) teve uma duração menor que o grupo COM (238 minutos). E ainda que, o grupo CGOC e COM sem ISC apresentaram uma duração média (143 e 235 minutos respectivamente) menor que aqueles com ISC (205 e 259 minutos, respectivamente). Percebeu-se, ainda, que a duração média entre os grupos sem ISC e os que tiveram a notificação da ISC pós-alta apresentaram uma duração média semelhante (Figura 2).

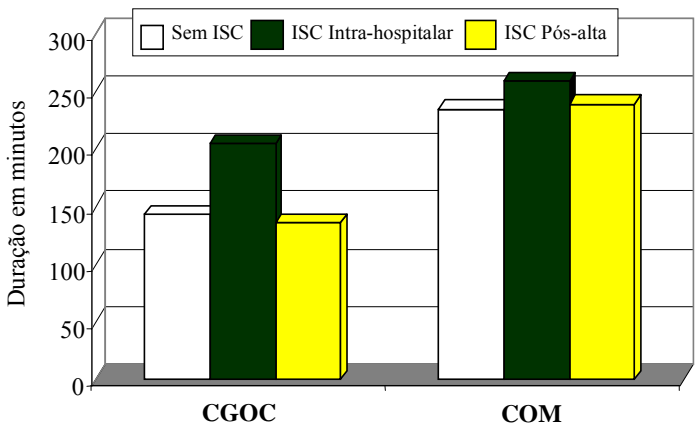

Figura 2 - Duração média dos procedimentos cirúrgicos dos grupos CCOC e COM de acordo com a ocorrência ou não da ISC - São Paulo - 2002

Para o intervalo de manifestação da ISC a Tabela 1 denota que no grupo CGOC durante a internação até o sétimo dia, o percentual encontrado foi de $16,7 \%$ e após a alta até o décimo quarto-dia esta foi de $76,9 \%$. Para o grupo COM as ISC detectadas durante a internação até o sétimo dia chegaram a $75 \%$ do total de casos e após a alta em quatorze dias de seguimento estas chegaram a $85,4 \%$.

Tabela 1- Distribuição da ISC nos grupos CGOC e COM de acordo com o intervalo de notificação - São Paulo - 2002

\begin{tabular}{|c|c|c|c|c|}
\hline \multirow{2}{*}{$\begin{array}{l}\text { Intervalo de tempo } \\
\text { (em dias) }\end{array}$} & \multicolumn{2}{|c|}{ CGOC } & \multicolumn{2}{|c|}{ COM } \\
\hline & Intra-hospitalar & Pós-alta & Intra-hospitalar & Pós-alta \\
\hline$<7$ & 1 & 3 & 3 & 20 \\
\hline$\geq 7 \mathrm{e}<14$ & 1 & 7 & 1 & 15 \\
\hline$\geq 14$ e $<21$ & 2 & 2 & - & 5 \\
\hline$\geq 21$ & 2 & 1 & - & 1 \\
\hline Total & 6 & 13 & 4 & 41 \\
\hline
\end{tabular}

Em relação à determinação do sitio específico da ISC, as mais freqüentes foram na categoria superficial tanto intrahospitalar como após a alta. Assim no grupo CGOC registrou-se na categoria superficial a totalidade da ISC diagnosticada intra-hospitalar, para aquelas detectadas após a alta, estas se distribuíram em 84,6\% superficial e 15,4\% profunda.
Para o grupo COM foram observadas $75 \%$ das ISC durante a internação na categoria superficial e $25 \%$ profunda. Após a alta nesse grupo constatou-se que $97,6 \%$ da ISC foi classificada como superficial e somente $2,4 \%$ como profunda. Nesse estudo não se observou nenhuma ISC como de órgão/cavidade (Tabela 2).

Tabela 2 - Distribuição da ISC nos grupos CGOC e COM segundo o local de notificação (intra hospitalar e após a alta) e sitio específico - São Paulo - 2002

\begin{tabular}{|c|c|c|c|c|c|}
\hline \multirow[t]{2}{*}{ Grupo } & \multicolumn{2}{|c|}{ Intra-hospitalar } & \multicolumn{2}{|c|}{ Pós-alta } & \multirow[t]{2}{*}{ Total } \\
\hline & Superficial & Profunda & Superficial & Profunda & \\
\hline $\mathrm{COM}$ & $3(75,0)$ & $1(25,0)$ & $40(97,6)$ & $1(2,4)$ & 45 \\
\hline CGOC & $6(100,0)$ & 0 - & $11(84,6)$ & $2(15,4)$ & 19 \\
\hline Total & $9(90,0)$ & $1(10,0)$ & $51(94,4)$ & $3(5,6)$ & 64 \\
\hline
\end{tabular}

Nota: Percentuais entre parêntesis 


\section{DISCUSSÃO}

Dentre as diversas especialidades cirúrgicas, uma grande preocupação se refere a taxa de incidência da ISC dos pacientes submetidos a cirurgia do aparelho digestivo (CAD) devido a ampla diversidade de potencial de contaminação de tais procedimentos, perfil dos pacientes, doença de base associada e ainda, por representar na maioria das instituições, o maior número de procedimentos cirúrgicos realizados. Geralmente esta especialidade se subdivide em diversos sub-grupos como parede (hérnias), estômago (esôfago e intestino delgado), fígado e vias biliares, intestino (coloproctologia), cirurgias por videolaparoscopia e transplante de órgãos.

Entretanto, a ênfase deste estudo se referiu àquelas realizadas em nível de estômago, como a cirurgia para a obesidade mórbida e as cirurgias gástricas por outras causas, gastrectomias incluindo-se os casos de câncer e as úlceras.

Sugerido pela própria denominação, a cirurgia para a obesidade é aquela decorrente de um quadro de obesidade mórbida, considerada uma doença da atualidade por atingir cada vez mais um maior número de pessoas no mundo, sendo em alguns países, pela sua alta prevalência, reconhecida como uma epidemia ${ }^{(15)}$.

Nos Estados Unidos, estudos estimam que a obesidade varie de $19,8 \%$ a $30,5 \%$ sendo que as diferenças encontradas podem estar baseadas na condução dos estudos em virtude dos critérios de mensuração de dados (dieta, atividade física e outros fatores difíceis de se ava-

Dentre as diversas especialidades cirúrgicas, uma grande preocupação se refere a taxa de incidência da ISC dos pacientes submetidos a cirurgia do aparelho digestivo

(CAD). liar) e auto-informação sobre o peso e altura. No Brasil, nas regiões Nordeste e Sudeste, $8 \%$ dos adultos são obesos (índice de massa corpórea igual a 30), sendo que destes, $70 \%$ são mulheres ${ }^{(15-18)}$.

Um grande avanço para o tratamento da obesidade mórbida constitui a realização de cirurgias específicas, que vão desde a passagem de um balão gástrico, que ocupa dois terços do estômago, até a derivação bílio-digestiva, além de outras técnicas que permitem o funcionamento do estômago em apenas $1 / 3$ de sua capacidade (de $1.500 \mathrm{ml}$ para $500 \mathrm{ml})^{(18)}$.

Assim, após os procedimentos cirúrgicos avaliados, a incidência da ISC detectada durante a internação de 5,0\% (4/81) em pacientes submetidos a COM e de 7,8\% (6/77) na CGOC é menor que a informada em estudos brasileiros de até $13 \% \%^{(5,8-9,12)}$.

Na vigilância pós-alta nesse estudo, verificou-se uma incidência da ISC no grupo COM de 50,6\% (41/81) e no grupo CGOC de 16,9\% (13/77). Situação essa, inversa a incidência da ISC detectada no período intra-hospitalar.
Diversos fatores podem estar explicando esta maior incidência da ISC após a alta no presente estudo.

Analisando-se a diferença entre os métodos utilizados para a vigilância pós-alta verifica-se que o retorno ambulatorial tem sido considerado como método de referência, associado a uma maior confiabilidade, apesar desta modalidade implicar em uma estrutura física e de recursos humanos diferenciada, o que nem sempre é possível para a maioria das instituições. Sua principal vantagem reside no fato de que, este tipo de vigilância propõe que todas as ISC sejam notificadas, independentes de sua localização. Isto se fundamenta na dificuldade observada de que, quando médicos realizam a notificação da ISC em seus consultórios/ ambulatórios, as superficiais geralmente deixam de ser notificadas, pois quase sempre não necessitam de terapia antimicrobiana, além de serem de fácil resolução, bastando a aplicação de calor local ${ }^{(5)}$.

O contato telefônico, realizado entre os pacientes submetidos a COM pode ser considerado como um método de fácil realização, de baixo custo, geralmente utilizado quando não é possível ao paciente retornar ao ambulatório do hospital. A sensibilidade pode ser uma limitação, pelo viés de informação, porém, como neste estudo foi realizado o contato por um profissional específico treinado e que direcionava as perguntas de forma objetiva e clara ao evento de interesse (presença de pus no local da incisão cirúrgica, febre, necessidade de retorno ao médico por alguma questão relacionada a cirurgia e/ou uso de antimicrobianos), acreditase que a possibilidade de viés de informação tenha sido controlada.

Em relação à alta proporção de ISC no grupo COM, pode se inferir que este grupo essencialmente formado por pacientes obesos, apresenta um importante fator de risco prévio a cirurgia. É descrito na literatura que pacientes obesos tem um risco aumentado e reconhecido para ISC ${ }^{(5,19-20)}$. O estudo ${ }^{(19)}$ é considerado uma importante referência para a comprovação de que a espessura do tecido adiposo tem influência direta e proporcional nas taxas de infecção. Neste estudo, em pacientes, onde a camada de tecido adiposo foi menor que 3,0 centímetros (cm), a taxa de infecção foi de $6,2 \%$, e em pacientes cuja espessura de tecido adiposo foi maior que $3,5 \mathrm{~cm}$, a taxa encontrada de ISC foi de 20,0\%. Esta forte associação, com a ocorrência da ISC parece estar relacionada ao fato de que o tecido adiposo é pouco vascularizado levando a procedimentos cirúrgicos mais demorados e à maior facilidade de trauma da parede abdominal. Por estes fatores, o autor concluiu que a exposição tecidual do paciente obeso é bem maior que o paciente não-obeso, com maiores possibilidades de formação de espaço morto e necessidades de utilização de suturas subcutâneas para fechá-los ${ }^{(19)}$. 
Por outro lado, o fato do seguimento deste grupo (COM) realizado por telefone não permitir a avaliação direta da ferida e a elevada taxa de ISC encontrada nesse grupo leva a pensar que uma das hipóteses para justificar esta elevada incidência pode ser atribuída à interpretação equivocada do paciente em relação ao material secretado pela ferida operatória, o seroma. O seroma, entretanto, constitui uma manifestação bastante comum no pós-operatório, principalmente desses pacientes, devido à espessa camada de tecido adiposo podendo levar o paciente a falsa idéia da presença de uma infecção ${ }^{(21-23)}$. Entretanto, apesar de tais considerações, ainda assim justifica-se o seguimento pós-alta, pois, no grupo da CGOC, constatou-se que de $24,7 \%$ da taxa de incidência global da ISC, $68,4 \%$ foram notificadas após a alta hospitalar, por avaliação direta do paciente.

Para as variáveis propostas no estudo (tempo de permanência, potencial de contaminação, tipo de cirurgia (eletiva/ urgência), duração da cirurgia, e ASA) como possíveis de explicar a associação das mesmas, com a ocorrência da ISC, foi realizada a análise univariada entre os grupos CGOC e COM e a presença ou não da ISC e o local onde a mesma foi detectada, não se observando nenhuma associação significativa.

Somente a variável duração da cirurgia apresentou uma distribuição média semelhante entre os grupos sem ISC e os que tiveram a notificação da ISC após a alta. Para muitos autores $^{(8-14)}$, o risco da ISC, é proporcional à duração do ato cirúrgico em si, ou seja, quanto maior a duração da cirurgia maior a possibilidade da ocorrência da ISC, pela maior exposição tecidual.

Em relação a classificação da ISC de acordo com o sitio específico, houve uma predominância de ISC do tipo super-

\section{REFERÊNCIAS}

1. Mangram AJ, Horan TC, Pearson ML, Silver LC, Jarvis WR. Guideline for prevention of surgical site infection, 1999. Hospital Infection Control Practices Advisory Committee. Infect Control Hosp Epidemiol. 1999;20(4):250-78.

2. Fernandes AT, Ribeiro Filho N, Oliveira AC. Infecções do sitio cirúrgico. In: Oliveira AC, Albuquerque CP, Rocha LCM. Infecções hospitalares: abordagem, prevenção e controle. Rio de Janeiro: Medsi; 2005. p. 93-123.

3. Nichols R. Preventing surgical site infections: a surgeon's perspective. Emerg Infect Dis. 2001;7(2):220-4.

4. Brasil. Ministério da Saúde. Agência Nacional de Vigilância Sanitária (ANVISA). Portaria n. 2.616/98. Dispões sobre o controle de IH em estabelecimentos de saúde [legislação na Internet] Brasília; 1998. [citado 2006 dez. 6]. Disponível em: http://www.anvisa.gov.br ficial tanto durante a internação como após a alta em ambos os grupos, não sendo notificada em nenhum momento, a ISC do tipo órgão/cavidade. Como já mencionado anteriormente, a ISC superficial, diagnosticada após a alta é muitas vezes ignorada, por não apresentar nenhuma limitação para o paciente, não necessitar re-internação e, acima de tudo, por ser de fácil resolução do ponto de vista clínico, o que gera uma sub-notificação para o serviço de controle de infecção hospitalar.

\section{CONCLUSÃO}

Neste estudo, durante a vigilância intra-hospitalar a incidência da ISC, no grupo CGOC (7,8\%) foi maior que a do grupo COM (5\%), verificando-se uma incidência global da ISC de $24,7 \%$ e $55,5 \%$ nos grupos CGOC e COM respectivamente, com a vigilância pós-alta. Ou seja, um impacto de três e 11 vezes mais nas taxas de notificação dos referidos procedimentos.

As diferenças observadas na vigilância pós-alta entre ambos os grupos podem ser parcialmente explicadas pela diferença do método utilizado, retorno ambulatorial (CGOC) e contato telefônico (COM). Entretanto, estas são similares àquelas informadas pelo $\mathrm{CDC}$.

Os resultados dessa pesquisa permitem às Comissões de Controle de Infecção Hospitalar reavaliar a importância do acompanhamento pós-alta a fim de melhorar a confiabilidade das taxas de incidência das ISC. E ainda, melhorar a qualidade dos cuidados com os pacientes e a compreensão epidemiológica da ISC, além de viabilizar a implementação de medidas direcionadas a sua prevenção e controle.
5. Oliveira AC. Controle de egresso cirúrgico: impacto na incidência da infecção de sítio cirúrgico em um hospital universitário [dissertação]. Belo Horizonte: Escola de Enfermagem, Universidade Federal de Minas Gerais; 1999.

6. Oliveira AC, Soares JL, Garcia CA, Scatena PD, D'Osvaldo L, Ciosak SI. Seguimento pós-alta do paciente cirúrgico: uma análise da importância da subnotificação da incidência da infecção do sítio cirúrgico. REME Rev Min Enferm. 2003;7(1):48-51.

7. Rodrigues MAG, Almeida GN. Infecções do sítio cirúrgico. In: Martins MA. Manual de infecção hospitalar: epidemiologia, prevenção e controle. $2^{\mathrm{a}}$ ed. Rio de Janeiro: Medsi; 2001. cap.16, p. 171-89.

8. Ferraz EM, Ferraz AA, Bacelar TS, Albuquerque HST, Vasconcelos MDM, Leão CS. Controle de infecção em cirurgia geral resultado de um estudo prospectivo de 23 anos e 42.274 cirurgias. Rev Colégio Bras Cir. 2001;28(1):17-25. 
9. Oliveira AC, Martins MA, Martinho GH. Estudo comparativo do diagnóstico da infecção do sítio cirúrgico durante e após a internação. Rev Saúde Pública. 2002;36(6):717-22.

10. Manian FA. A surveillance of surgical site infections in alternative settings: exploring the current options. Am J Infect Control. 1997;25(1):102-5.

11. Medina-Cuadros M, Sillero-Arenas M, Martinez-Gallego G, Delgado-Rodriguez M. Surgical wound infection diagnosed after discharge from hospital: Epidemiologic differences with inhospital infections. Am J Infect Control. 1996;24(6):421-8.

12. Oliveira AC, Ciosak SI. Infecção de sítio cirúrgico no seguimento pós-alta: impacto na incidência e avaliação dos métodos utilizados. Rev Esc Enferm USP. 2004;38(4):379-85.

13. Poveda VB, Galvão MC, Hayashida M. Análise dos fatores de risco relacionados a incidência de infecção dos sitio cirúrgico em gatrocirurgias. Rev Esc Enferm USP. 2003;37(1):81-9.

14. Starling CEF, Pinheiro SMC, Couto BRGM. Vigilância epidemiológica das infecções hospitalares na prática diária, ensaios. Belo Horizonte: Cutiara; 1993.

15. Lehmann ALF, Valezi AC, Brito EM, Marson AC, Souza JCL. Correlation of gallbladder hipomotility and gallstone formation after bariatric surgery. Rev Col Bras Cir. 2006;33(5):285-8.

16. Mokdad AH, Serdula MK, Dietz WH, Bowman BA, Marks JS, Koplan JP. The spread of the obesity epidemic in the United States, 1991-1998. JAMA. 1999;282(16):1519-22.
17. Flegal KM, Carroll MD, Ogden CL, Johnson CL. Prevalence and trends in obesity among US adults, 1999-2000. JAMA. 2002;288(14):1723-7.

18. Freitas ACT, Freitas DT, Parolin MB, Campos ACL, Coelho JCU. Nonalcoholic fatty liver disease: evolution after gastric bypass. Arq. Gastroenterol. 2007;44(1):49-53.

19. Nystrom AS, Jonstam A, Höjer H, Ling L. Incisional infection after colorectal surgery in obese patients. Acta Chir Scand. 1987;153(3):225-7.

20. Grinbaum RS. Infecções do sitio cirúrgico e antibioticoprofilaxia em cirurgia. In: Rodrigues EAC, Mendonça JS, Amarante JMB, Alves Filho MB, Grinbaum RS, Ritchmann R. Infecções hospitalares: prevenção e controle. São Paulo: Sarvier; 1997. p. 149-61.

21. Rodriguez MD, Ortega AG, Arenas MS, Llorca J. Epidemiology of surgical-site infections diagnosed after hospital discharge: a prospective cohort study. Infect Control Hosp Epidemiol. 2001;22(1):24-30

22. Stockley JM, Allen RM, Thomlinson DF, Constantine CE. A district general hospital's method of post-operative infection surveillance including post-discharge follow-up, developed over a five-year period. J Hosp Infect. 2001;49(1):48-54.

23. Whitby M, McLaws ML, Collopy B, Looke DFL, Doidge S, Henderson B, et al. Post-discharge surveillance: can patients reliably diagnose surgical wound infections? J Hosp Infect 2002;52(3):155-60. 